Service social

\title{
Vieillir à travers le monde, contribution à une gérontologie comparée, sous la direction de Renaud Santerre et Georges Létourneau, Québec, Presses de l’Université Laval, 1989, 350 pages.
}

\section{Nicole Boucher}

Volume 40, numéro 1, 1991

Éthique et intervention sociale

URI : https://id.erudit.org/iderudit/706518ar

DOI : https://doi.org/10.7202/706518ar

Aller au sommaire du numéro

Éditeur(s)

École de service social de l'Université Laval

ISSN

1708-1734 (numérique)

Découvrir la revue

Citer ce compte rendu

Boucher, N. (1991). Compte rendu de [Vieillir à travers le monde, contribution à une gérontologie comparée, sous la direction de Renaud Santerre et Georges Létourneau, Québec, Presses de l’Université Laval, 1989, 350 pages.] Service social, 40(1), 143-144. https://doi.org/10.7202/706518ar d'utilisation que vous pouvez consulter en ligne. 


\title{
RECENSIONS
}

\author{
Vieillir à travers le monde, contribution à une gérontologie \\ comparée, sous la direction de Renaud SANTERRE et Georges \\ LÉtOURNeAu, Québec, Presses de I'Université Laval, 1989, \\ 350 pages.
}

Cet ouvrage collectif est le résultat de la collaboration d'une trentaine de personnes, dont celle de sept étudiantes de maîtrise en anthropologie qui mérite d'être particulièrement soulignée. Conçu comme un manuel d'anthropologie de la vieillesse, il a le mérite de rassembler des articles de base déjà parus ailleurs pour une moitié (Santerre, Arcand, Guemple, Biesele et Howell, Evans-Pritchard, Guillemard, Bergman, Fadel-Girgis, Kumagai, Forest et Streit, Corin et al.), et des textes originaux pour l'autre moitié. Une bibliographie générale de plus de 400 titres complète avantageusement les textes.

Étant donné l'abondance du matériel, nous attirons votre attention sur certains textes originaux pour chacune des trois parties du livre. L'ensemble de ces productions se situent en rapport avec l'une des contributions théoriques majeures de l'anthropologie à l'étude du vieillissement, soit la théorie du vieillissement et de la modernisation. Elles permettent en première partie d'aborder des questions de méthodologie comparative (quantitative vs qualitative); en deuxième partie sont rassemblées 16 études de cas et, finalement, en troisième partie, on tente de dégager des facteurs généraux conditionnant le vieillissement.

La partie méthodologique débute par un texte de Georges Létourneau de I'Université de Montréal sur l'étude comparative du vieillissement. À partir d'une vision large de l'anthropologie comme "étude de l'ensemble des facteurs qui affectent la condition de vie des êtres humains de tous les temps et dans tous les lieux ", I'auteur propose une approche systémique permettant de tenir compte de plusieurs ordres de facteurs susceptibles d'affecter les conditions de vie des personnes âgées dans une société. Ensuite, il présente succinctement différentes méthodes d'études comparatives développées en anthropologie soit pour définir la vieillesse, soit pour découvrir comment le vieillissement s'organise au sein de différents groupes dans les sociétés complexes, soit pour comparer des trajectoires de vie et des stratifications sociales par âge.

L'article de Renaud Santerre, pour sa part, porte sur les études utilisant principalement des techniques de méthodologie qualitative, I'observation participante, l'histoire de vie et l'étude des communautés.

II est impossible ici de rendre compte de toute la richesse des études de cas de la deuxième partie. Ces études concernent les Inuit, le Québec, les États-Unis, I'Union soviétique, la France, le Japon, le Portugal, I'Inde, Samoa, 
l'Égypte, Israël et le Maroc. Chaque étude permet de mettre en évidence des processus différents de construction culturelle de la vieillesse.

La dernière partie sur les facteurs généraux conditionnant le vieillissement s'appuie nettement sur "le concept de vieillissement différentiel " traité dans I'article de Francis Forest et Ursula Streit. L'article original de Renaud Santerre, "Ethnicité et vieillesse québécoise ", nous donne des indications statistiques de base sur le vieillissement dans les 22 principaux groupes ethniques composant la mosaïque québécoise.

En conclusion, nous considérons ce livre non seulement comme un excellent manuel pour les étudiants en gérontologie et en anthropologie, mais également comme une contribution importante de l'anthropologie québécoise à l'étude du vieillissement. Ces articles démontrent nettement l'importance de relativiser, différencier "les vieillesses " dans le temps et dans l'espace. On ne peut que féliciter les Presses de l'Université Laval d'avoir choisi ce document pour lancer une série en gérontologie. La qualité de l'œuvre de lancement est prometteuse pour une série d'excellence.

Nicole Boucher

École de service social

Université Laval

La créativité dans l'action sociale locale. Supplément au numéro 274-275 de la revue Correspondance Municipale regroupant les textes préparatoires au Troisième Colloque de Montrouge, organisé par l'Institut de travail social et de recherche sociale, à Montrouge, les 11, 12 et 13 mars 1987. Janvier-février 1987, 66 pages.

Quoique non récent, ce numéro spécial de la revue française Correspondance Municipale mérite toute notre attention, car il nous permet de saisir rapidement l'évolution du service social français à travers l'impact de la décentralisation sur les politiques sociales et la pratique des travailleurs sociaux (assistants sociaux plutôt) en France. En cette période de réforme des sèrvices sociaux québécois axée sur l'action locale, l'expérience française peut éventuellement nous aider à en comprendre les enjeux.

Tout au long des 15 articles de la revue, nous découvrons expériences, débats, analyses et prospectives sur les nouveaux objectifs et les nouvelles stratégies de l'action sociale française. L'enjeu du renouvellement de l'action est l'affaire de tous. Or, cette créativité réclamée suppose non seulement plus de flexibilité des institutions, mais également la mutation des fonctions professionnelles, des outils de management social et des méthodes d'intervention sociale.

Ainsi l'article de Brigitte Bouquet, directrice de l'école de Montrouge, qui nous situe rapidement l'ensemble des partenaires sociaux, nous explique à partir des réponses à un questionnaire les changements survenus après la décentralisation de 1982-1983. Nous y décelons que, à la suite de la promotion de l'action sociale locale, les rapports entre l'État, les départements et les communes sont souvent source de conflits surtout quant aux tâches des intervenants et à la répartition des ressources matérielles et professionnelles. Ainsi, 